\title{
Exploring health-related quality of life in eating disorders by a cross-sectional study and a comprehensive review
}

\author{
Monica Baiano ${ }^{1}$, Pierandrea Salvo ${ }^{1}$, Pierluigi Righetti ${ }^{1}$, Lucia Cereser ${ }^{1}$, Erika Baldissera ${ }^{1}$, Ilenia Camponogara ${ }^{1}$ \\ and Matteo Balestrieri ${ }^{2,3^{*}}$
}

\begin{abstract}
Background: People with eating disorders (ED) often report poor health-related quality of life (HRQoL), which is explicitly correlated to illness' severity and its effects on cognitive performance. We aimed to analyze health-related quality of life (HRQoL) in subgroups of eating disorder (ED) patients by using the brief version of WHOQoL questionnaire (WHOQoL-BREF) before treatment administration. Moreover, in order to compare our findings with other published data, we carried out a comprehensive review of the literature on HRQOL in ED patients.

Methods: Our review was carried out by means of an accurate data mining of PsychInfo and Medline databases and other available sources. In our cross-sectional study, eighty female ED patients (26 with bulimia nervosa, 33 with anorexia nervosa, 7 with binge eating disorder and 14 with ED not otherwise specified) completed the WHOQOL-BREF. HRQOL scores were compared among ED subgroups and clinical information (presence of previous contacts, length of illness, psychiatric comorbidity) was considered in the analysis.

Results: Our review shows that with few exceptions ED patients have a poorer HRQoL than the healthy population of control and sometimes the mental component of HRQOL is the most involved dimension. Moreover, there are no differences in the HRQoL among ED groups, even if AN patients in some studies have a lower HRQoL scores. Furthermore, BED patients have a poorer HRQoL than obese patients who do not have binge episodes. Finally, all treatments were positively correlated with an improvement on general and specific QoL dimensions. In our sample, ED subgroups differed only for Psychological Health HRQoL scores $(F=4.072, d f=3 ; p=0.01)$. No differences were found between inpatients and outpatients, treatment naive and previously treated patients and patients with or without psychiatric comorbidity. Moreover, HRQoL scores were not correlated to length of illness within each ED subgroup.

Conclusions: The analysis of the literature adds some relevant information on HRQoL in ED and may address the future research toward the exploration of specific questions. One of these may be the prominent role of Psychological Health domain in HRQoL, since our study confirms that this component is able to differentiate eating disorders.
\end{abstract}

Keywords: Eating disorders, Anorexia nervosa, Bulimia nervosa, Binge eating, HRQoL, Quality of life, WHOQoL-BREF, SF-36, Review

\footnotetext{
* Correspondence: matteo.balestrieri@uniud.it

${ }^{2}$ Unit of Psychiatry, Dept. of Experimental and Clinical Medical Sciences,

University of Udine, Udine, Italy

${ }^{3}$ Clinica Psichiatrica, Azienda Ospedaliero-Universitaria, P.le S.M. Misericordia

15, 34070 Udine, Italy

Full list of author information is available at the end of the article
} 


\section{Background}

According to the World Health Organization (WHO), QoL refers to "the individuals' perceptions of their position in life in the context of the culture and value systems in which they live and in relation to their goals, expectations, standards and concerns" [1]. On the other hand, the concept of health-related QoL (HRQoL) encompasses those aspects of overall quality of life that can be clearly shown to affect health, either physical or mental. On the individual level, this includes physical and mental health perceptions and their correlates, while on the community level HRQoL includes resources, conditions, policies, and practices that influence a population's health perceptions and functional status [2]. As reviewed by Engel and colleagues [3], most of studies investigating the HRQoL in eating disorder (ED) patients measured the effects of abnormal eating behavior on health status but not on HRQoL.

Specifically, the HRQoL is associated with the degree of illness-related disability and effects of medical interventions on perceived HRQoL [4,5]. Indeed, people with ED often report poor HRQoL [6], which is explicitly correlated to illness' severity and its effects on cognitive performance $[7,8]$. Cognitive impairments may significantly reduce adherence to individualized rehabilitation programs, thus favoring chronicity and psychosocial deterioration [9]. Moreover, ED are frequently associated with comorbid psychiatric disorders, especially anxiety, somatoform and depressive disorders, which further prevent recovery and increase the probability of resistance to treatment efforts [10,11].

Hence, the assessment of HRQoL in ED is crucial to predict the clinical outcome in patients undergoing specific treatments, as well as the risk of relapse and recurrence [12]. Although some ED-specific instruments have been used to assess HRQoL [13], none of them offers a global viewpoint of patient's perception of HRQoL. Conversely, the World Health Organization Quality of Life (WHOQoL) questionnaire [1] measures patient's subjective awareness of illness related to physical and psychosocial dysfunctioning.

We wanted to investigate HRQoL in eighty female ED patients attending a Centre for Eating and Weight Disorders in Italy. In order to place our results within the frame of the current knowledge, we analyzed the literature and realized that a comprehensive review on the factors that can modulate HRQoL in eating disorders was lacking. So, we decided to implement a review on this topic, by selecting published papers written in English. The aims of the review were to:

- test the hypothesis that HRQoL is poorer in ED subjects than in healthy individuals

- explore the existence of different levels of HRQoL among different ED
- explore whether ED subjects have different impairments on mental vs. physical dimension of HRQoL

- explore the existence of differences in HRQoL between obese subjects and subjects with BED

- analyze the efficiency of ED-specific HRQoL instruments as compared with more general instruments

- analyze the efficacy on HRQoL of the treatment proposed for ED, including bariatric surgery.

Once that this review was completed, we were able to compare the results obtained in our sample with those emerging from all other studies. We were specifically interested in analyzing the HRQoL among patients with different eating disorders at the beginning of a psychonutritional rehabilitation program and to explore whether comorbid DSM-IV diagnoses, setting of care and history of previous treatment were involved in their perception of HRQoL.

\section{Methods}

\section{Analysis of the literature}

We firstly consulted other reviews on this topic, which revealed a number of shortcomings. The review by Engel et al. [3] is a narrative review focused only on the instruments used to measure the HRQoL in ED patients. The Authors identified four ED-specific HRQoL instruments (the 25-item EDQOL, the 40-item EDQLS, the 55-item HeRQUoLED and the 20-item QOL-ED) and discussed their relevance in the field of the treatment of ED.

A second review was carried out by Passarelli-Tirico et al. [14], who classified the papers according to the quality of the design, as defined by the Australian governative Agency NHMRC. The shortcomings of this review are that it is not in English and does not include the results of HRQoL analyses.

The third review was carried out by Jenkins et al. using a narrative approach [15]. This is a comprehensive review, which allows to enter into the details of the papers and to analyze specific issues such as the HRQoL associated with subclinical ED pathology, BMI, or purging and other compensatory behaviors. However, it does not include a general table to compare the studies and does not attempt to systematically describe the research design, the setting or the type of assessment used.

In our comprehensive review we aimed to include papers that used validated HRQoL instrument and to classify patients according to definite diagnoses. Since this was not always possible, we considered also papers that assessed the patients according to eating dimensions or to subclinical levels of disordered eating.

Accurate data mining was conducted by consulting PsychInfo and Medline databases and other available 
sources, such as the references included in the papers reviewed. The databases were examined thoroughly using the keywords "quality of life, QoL, HRQoL, functional impairment, eating disorders, anorexia nervosa, bulimia nervosa, binge eating disorder".

The accuracy of the assessment among the papers included was quite variable, ranging from simple ED questionnaires to clinical or semi-structured interviews. Most frequently, the assessment was conducted with instruments of the Eating Disorders Examination (EDE) series, either questionnaire or interview. Other structured instruments were the SCID or the PRIME-MD. In reporting the data means and SD where not shown, since these statistics were not always inferable (for example, they were shown in figures and not in tables) or else were too many (one statistic for each ED and for each subscale) to be included in the table. Moreover, apart from studies using SF-36, most statistics were taken with miscellaneous instruments and thus were not comparable.

Finally we excluded those papers that analyzed the HRQoL in obese patients without attempting to assess the presence of a whatsoever ED within the sample.

\section{Procedures of the cross-sectional study}

We recruited eighty female ED patients (mean age: $28.24 \pm 11.28$ SD; range: $13-61$ years) attending the Centre for Eating and Weight Disorders of the local Socio-Health Unit (ASSL "Veneto Orientale", Portogruaro, Italy). This Center is a highly specialized unit, where treatment-resistant ED subjects are referred from a vast area including health districts both of Veneto and of Friuli regions. On the other hand also subjects living in the nearby surroundings can be accepted. Patients' diagnoses were established using the DSM-IV-TR criteria for Anorexia Nervosa, Bulimia Nervosa, and Binge Eating Disorder. Subjects who did not meet full diagnostic criteria were classified as Eating Disorder Not Otherwise Specified. Comorbidities were assessed taking in consideration the medical history, and confirming their current presence with the clinical assessment. Exclusion criteria included the presence of clinically relevant multiorganic disorder or cerebral organic impairment and patients not completing the assessment for language barriers. All patients were informed about the main objectives of our search and all signed written informed consent.

Our study was carried out in compliance with the Helsinki Declaration, using the database of Centre for Weight and Eating Disorders of Portogruaro, a credited agency of the Regional Health System. Patients gave their written consent to the use of their data for both clinical and research purposes at the time of their first contact with the Centre. Since no new instrument or investigation were used, a specific request to the local ethics committee was not advanced, being valid the principle of the availability for research of the data gathered in the clinical practice.

All participants were assessed for self-perception of HRQoL using the WHOQoL-BREF [1] at the beginning of treatment. This questionnaire is a 26 -item short version of the WHOQoL scale measuring the subjective perception of quality of life associated with physical and psychological health, social performance and environment's characteristics. The Physical Health scale specifically investigates activity of daily living, dependence on drugs and medical supports, level of energy, mobility, pain and discomfort, sleep and rest, work capacity. The Psychological Health scale explores body image and appearance, positive and negative feelings, self-esteem, spirituality and personal believes, cognitive functioning. The Social Relationship scale measures quality of social relationships, social support and sexual activity. Finally, the Environment scale evaluates individual's socio-economic conditions (as financial resources, freedom, physical safety and security) and the availability of facilities in living and working context (accessibility to health and social care, home environment, chances for acquiring new information and skills, recreation and leisure activities, physical environment and transport).

Items are scored on a Likert five-point scale, were " 1 " means severe discontent and " 5 " great approval; patients had to evaluate their quality of life considering the two weeks preceding the administration of the questionnaire. The mean score of items within each scale was multiplied by 4 in order to be comparable to those of the 100-items WHOQOL scale.

Finally, height and weight were measured to calculate the body mass index (BMI: $\mathrm{kg} / \mathrm{m}^{2}$ ).

\section{Statistical analysis}

Data were analyzed using SPSS Version 16.0 for Windows [16] and STATA 10.0 [17]. The statistical significance (alpha) was set at $\mathrm{p}<0.05$. Firstly, data were inspected for normality using the Shapiro-Wilk test.

Socio-demographic and clinical variables were compared among ED subgroups using the Kruskal-Wallis or Fisher's exact test, as appropriate. Finally, an analysis of covariance (multivariate ANCOVA) was used to examine whether 1) there were significant differences between ED subgroups, after controlling for age and length of illness; 2) there were differences for HRQoL scores between ED inpatients and outpatients, ED patients with and without history of previous treatment, patients with and without comorbid DSM-IV diagnosis, controlling for ED diagnosis. Bonferroni's correction for multiple comparisons was also applied.

Finally, Pearson's correlation analysis was performed to explore the relationship between HRQoL scores and length of illness. 


\section{Results}

As it shown in Table 1, three main typology of study could be identified in the literature. The first one is the population survey, carried out with different methods and with dissimilar response rates. Some were postal surveys, others were conducted via telephone, and further more used a face to face interview. A second type of design is the cross-sectional analysis of samples of subjects attending outpatients ED centers or of patients waiting for a gastric by-pass surgery. The third type of design is the cohort prospective study. Within this category, there is one single multi-wave survey and a number of papers focused on treatments: within the latter studies, we can enumerate RCT studies with drugs, one study with a specific psychotherapy (CBT) treatment, studies on patients who attended a nutrition program or other unspecified programs in ED centers and one study which evaluated a gastric by-pass surgery intervention.

An important issue is the preference accorded from most studies to the SF-36 scale or its derivatives for the assessment of HRQoL. The SF-36 is a well-known but unspecific instrument for the study of ED and allows a distinction between mental component symptoms (MCS) and physical component symptoms (PCS). Other generic instruments include for example the WHO scale WHOBREF, which was used in a few studies. More specific instruments are EDQoL, the HeRQUoLED, the EDQLS, the IWQOL, but only few studies adopted them.

The main evidence that comes out from our review is that with few exceptions ED patients have a poorer HRQoL than the healthy population of control. Frequently MCS is the most involved dimension, which means that ED patients are particularly vulnerable to impairment in the QoL because of their psychic difficulties, being the physical component less harming. But this is not always the rule.

Another piece of evidence is that frequently there are no differences in the HRQoL among ED groups. AN patients in some studies have a lower HRQoL and this is particularly evident when a specific instrument is used, like the EDQoL.

Among potential surgical patients waiting for a GBP, most - but not all - studies conclude that BED patients have a poorer HRQoL than obese patients who do not have binge episodes.

Finally, when we consider the efficacy of the treatment on HRQoL measures, all treatments were positively correlated with an improvement on the general and, when examined, specific QoL dimensions. However, when compared with healthy individuals, their HRQoL remained still below the norm.

\section{Characteristics of our sample}

All relevant socio-demographic and clinical data of our cross-sectional study are reported on Tables 2 and 3 .
Fifty-nine out of 80 subjects were outpatients (73.8\%) whereas the remaining 21 (26.3\%) were inpatients. Among all patients, 33 (41.3\%) were affected by AN (mean age: 26.1 years $\pm 10.6 \mathrm{SD}), 26$ (32.5\%) were affected by $\mathrm{BN}$ (mean age: 27.5 years $\pm 8.0 \mathrm{SD}$ ), 7 were diagnosed as BED subjects $(8.8 \%)$ (mean age: 41.6 years $\pm 14.0 \mathrm{SD}$ ) and 14 patients suffered from EDNOS (17.5\%) (mean age: 28.1 years $\pm 13.3 \mathrm{SD}$ ). In addition, 14 patients (11 inpatients and 3 outpatients) fulfilled DSM-IV criteria for other ED: two for Borderline Personality Disorder (2.5\%), nine for Major Depressive Disorder (11.3\%) and one each for Bipolar Disorder, Alcohol Abuse and Social Phobia. Forty-six patients had a history of previous therapies for ED (25 inpatients and 21 outpatients) whereas the remaining 34 subjects were all treatment-naïve ED inpatients.

ED subgroups significantly differed for age $\left(\chi^{2}=8.293\right.$, $\mathrm{p}=0.040)$, BMI $\left(\mathrm{X}^{2}=54.916 ; \mathrm{p}<0.001\right)$, age at onset $\left(x^{2}=; p=0.02\right)$, age at first evaluation $\left(x^{2}=8.198 ; p=0.04\right)$ and length of illness $\left(x^{2}=9.585 ; p=0.022\right)$. Also, differences among ED subgroups were found for marital status $(\mathrm{p}<0.001)$, setting $(\mathrm{p}=0.005)$, history of previous treatments $(\mathrm{p}<0.001)$ but not for educational level or living conditions, occupational status, proportion of patients completing their studies and DSM-IV psychiatric disorder comorbidity $(p>0.05)$.

Significant differences emerged when considering the presence of a history of previous treatments for ED, both between ED inpatients (100\% untreated) and outpatients (54.3\% untreated) $(\mathrm{p}<0.001)$ and between patients with psychiatric comorbidity (14.2\% untreated) and without psychiatric comorbidity $(48.5 \%$ untreated $)(\mathrm{p}=0.03)$. In opposition, no difference was detected for psychiatric comorbidity between inpatients ( $81 \%$ no comorbidity) and outpatients (85.7\% no comorbidity) ( $\mathrm{p}>0.05)$.

\section{HRQoL scores in our sample}

All ED patients' HRQoL scores were below normative population values (Table 4). Results of ANCOVA showed that ED subgroups differed only for Psychological Health QoL scores $(\mathrm{F}=4.072$, d.f. $=3 ; \mathrm{p}=0.01$; ANCOVA, age and length of illness as covariates). No differences were found for all HRQoL scores when patients were subgrouped for BMI levels (underweight: < $18.5 \mathrm{BMI}$; normal weight: 18.5-24 BMI; overweight/obese: $\geq 25)(p>0.05$, ANOVA or Kruskal-Wallis test, as appropriate). As to respect to illness' awareness, differences among ED subgroups did not reach statistical significance ( $p>0.05$, Fisher's exact test).

No differences for HRQoL scores were found between inpatients and outpatients, as well as treatment naïve or previously treated patients $(\mathrm{p}>0.05)$. Also, ED patients with a DSM-IV comorbid diagnosis did not differ from those without psychiatric comorbidity for HRQoL scores ( $p>0.05$; ANCOVA, ED diagnosis as covariate). Ultimately, 
Table 1 Review of the literature on QoL in eating disorders subjects

\begin{tabular}{|c|c|c|c|c|c|c|c|}
\hline Study & Design, treatment & $\begin{array}{l}\text { Setting, characteristics } \\
\text { of whole sample }\end{array}$ & ED pts studied & $\begin{array}{l}\text { Control or reference } \\
\text { group(s) }\end{array}$ & Assessment & $\begin{array}{c}\text { HRQoL } \\
\text { Instrument }\end{array}$ & $\begin{array}{c}\text { Results on general and specific } \\
\text { HRQoL measures (> means } \\
\text { superior QoL) }\end{array}$ \\
\hline \multicolumn{8}{|l|}{ Surveys } \\
\hline \multirow{2}{*}{$\begin{array}{l}\text { [12] Spitzer } \\
\text { et al., } 1995\end{array}$} & \multirow[t]{2}{*}{ Two-stage survey } & \multirow[t]{2}{*}{1000 primary care pts } & \multirow{2}{*}{$\begin{array}{l}30 \text { BED (with } 84 \% \\
\text { psychiatric comorbidity) }\end{array}$} & \multirow{2}{*}{$\begin{array}{l}614 \text { without Mental } \\
\text { Disorders (MD) }\end{array}$} & \multirow[t]{2}{*}{ PRIME-MD } & \multirow[t]{2}{*}{ SF-20 } & - BED (as well other MD) < No-MD. \\
\hline & & & & & & & $\begin{array}{l}\cdot \text { BED }<\text { non-BED on social } \\
\text { functioning and bodily pain }\end{array}$ \\
\hline [18] Hay 2003 & Comunity survey & $\begin{array}{l}3010 \text { out of } 4400 \\
\text { (response rate } 70 \% \text { ) }\end{array}$ & $\begin{array}{l}78 \text { BED, } 60 \text { subj with } \\
\text { extreme weight control } \\
\text { behaviors (EWCB) }\end{array}$ & $\begin{array}{l}\text { Australian normative } \\
\text { sample }\end{array}$ & EDE & $\begin{array}{l}\text { SF-36, } \\
\text { AQoL }\end{array}$ & $\begin{array}{l}\cdot \text { BED and EWCB }<\text { Normative } \\
\text { on MCS }\end{array}$ \\
\hline \multirow[t]{2}{*}{$\begin{array}{l}\text { [19] Doll } \\
\text { et al., } 2005\end{array}$} & \multirow[t]{2}{*}{ Postal survey } & \multirow[t]{2}{*}{$\begin{array}{l}1439 \text { out of } 3750 \text { students } \\
\text { (response rate } 42 \% \text { ) }\end{array}$} & \multirow{2}{*}{$\begin{array}{l}83 \text { (5.8\% of } \\
\text { respondents) with ED } \\
\text { (54 BN, } 22 \mathrm{BED}, 7 \mathrm{AN})\end{array}$} & \multirow[t]{2}{*}{1148 non-ED subjects } & \multirow[t]{2}{*}{$\begin{array}{l}\text { Ad-hoc questionnaire } \\
\text { based on DSM-IV }\end{array}$} & \multirow[t]{2}{*}{ SF-36 } & $\begin{array}{l}\cdot \text { ED }<\text { non-ED subj on MCS, } \\
\text { but not on PCS. }\end{array}$ \\
\hline & & & & & & & $\begin{array}{l}\text { - BN and BED }<\text { non-ED subj } \\
\text { on MCS }\end{array}$ \\
\hline $\begin{array}{l}\text { [20] Herpetz- } \\
\text { Dahlmann } \\
\text { et al., } 2008\end{array}$ & Community survey & 1895 adolescents & $\begin{array}{l}400 \text { ED not classified } \\
\text { according DSM criteria }\end{array}$ & 1495 non-ED & $\begin{array}{l}\text { SCOFF, not confirmed } \\
\text { by interview }\end{array}$ & KINDL-R & $\cdot E D<$ non-ED \\
\hline $\begin{array}{l}\text { [21] Vallance } \\
\text { et al., } 2011\end{array}$ & Cross-sectional study & $\begin{array}{l}\text { Recruitment at the } \\
\text { university campus and } \\
\text { in newspapers }\end{array}$ & $\begin{array}{l}103 \text { women with } \\
2+\text { episodes of binge } \\
\text { eating per month }\end{array}$ & $\begin{array}{l}109 \text { women with }<2 \\
\text { episodes of binge } \\
\text { eating per month }\end{array}$ & EDE-Q, EDI-2 & SF-36 & $\begin{array}{l}\text { - High frequency of binge eating } \\
\text { predicted poorer QoL }\end{array}$ \\
\hline $\begin{array}{l}\text { [22] Mond } \\
\text { et al., } 2012\end{array}$ & $\begin{array}{l}\text { Two stage community } \\
\text { study }\end{array}$ & $\begin{array}{l}324 \text { interviewed at the } \\
\text { second stage }\end{array}$ & $\begin{array}{l}159 \mathrm{ED} \text { (30 BN, } 20 \text { BED, } \\
109 \text { EDNOS) }\end{array}$ & $\begin{array}{l}232 \text { healthy women } \\
\text { from different survey } \\
\text { in same area }\end{array}$ & $\mathrm{EDE}-\mathrm{Q}+\mathrm{EDE}$ & $\begin{array}{l}\text { SF- 12, } \\
\text { WHOQOL- } \\
\text { BREF }\end{array}$ & $\cdot$ ED $<$ Healthy women on MCS \\
\hline \multirow[t]{3}{*}{$\begin{array}{l}\text { [23] Mitchison } \\
\text { et al., } 2013\end{array}$} & \multirow[t]{3}{*}{ Population survey } & \multirow[t]{3}{*}{$\begin{array}{l}3034 \text { out of } 5000 \text { selected } \\
\text { (response rate } 60.7 \% \text { ) }\end{array}$} & \multirow[t]{3}{*}{$\begin{array}{l}89 \text { AN }(2.9 \% \text { of } \\
\text { respondents) }\end{array}$} & \multirow[t]{3}{*}{$\begin{array}{l}2945 \text { subj with no } \\
\text { history of AN }\end{array}$} & \multirow[t]{3}{*}{$\begin{array}{l}\text { Interview based } \\
\text { on } \mathrm{EDE}\end{array}$} & \multirow[t]{3}{*}{ SF-36 } & $\begin{array}{l}\text { - AN < other subj on most } \\
\text { domains, including MCS. }\end{array}$ \\
\hline & & & & & & & $\begin{array}{l}\text { - Subj with history of AN < other } \\
\text { subj on MCS but not on PCS. }\end{array}$ \\
\hline & & & & & & & $\begin{array}{l}\text { - Impairment on social functioning } \\
\text { and role limitations greater with } \\
\text { current ED symptoms }\end{array}$ \\
\hline \multicolumn{8}{|l|}{$\begin{array}{l}\text { Cross-sectional } \\
\text { studies }\end{array}$} \\
\hline \multicolumn{8}{|l|}{ ED patients } \\
\hline $\begin{array}{l}\text { [24] Keilen } \\
\text { et al., } 1994\end{array}$ & Cross-sectional study & ED outpatient center & 126 ED (52 AN, 74 BN) & $\begin{array}{l}98 \text { males with angina; } \\
122 \text { hearth transplant } \\
\text { candidates; } 54 \text { cystic } \\
\text { fibrosis pts; } 91 \text { students }\end{array}$ & $\begin{array}{l}\text { Clinical interview } \\
\quad \text { (DSM-III-R) }\end{array}$ & NHP & $\begin{array}{l}\text { - Specific differences between ED } \\
\text { and pts with organic diseases }\end{array}$ \\
\hline \multirow{2}{*}{$\begin{array}{l}\text { [10] Padierna } \\
\text { et al., } 2000\end{array}$} & \multirow[t]{2}{*}{ Cross-sectional study } & \multirow[t]{2}{*}{ ED outpatient center } & \multirow{2}{*}{$\begin{array}{l}197 \mathrm{ED}(116 \mathrm{AN}, \\
64 \mathrm{BN}, 17 \mathrm{BED})\end{array}$} & \multirow{2}{*}{$\begin{array}{l}\text { Norm-based scoring of } \\
\text { Spanish general } \\
\text { population }\end{array}$} & \multirow{2}{*}{$\begin{array}{l}\text { Clinical interview } \\
\text { (DSM-IV) }\end{array}$} & \multirow[t]{2}{*}{ SF-36 } & - ED pts < normative population. \\
\hline & & & & & & & $\begin{array}{l}- \text { BED }<\text { other ED on physical } \\
\text { functioning }\end{array}$ \\
\hline
\end{tabular}


Table 1 Review of the literature on QoL in eating disorders subjects (Continued)

\begin{tabular}{|c|c|c|c|c|c|c|c|}
\hline \multirow[t]{2}{*}{$\begin{array}{l}\text { [25] Gonzalez- } \\
\text { Pinto et al., } 2004\end{array}$} & \multirow[t]{2}{*}{ Cross-sectional study } & \multirow[t]{2}{*}{ ED outpatient center } & \multirow[t]{2}{*}{$47 \mathrm{AN}$} & \multirow[t]{2}{*}{ No control } & \multirow[t]{2}{*}{ SCID I and II } & \multirow[t]{2}{*}{ SF-36 } & $\begin{array}{l}\text { - Predictive variables for PCS: poor } \\
\text { outcome in previous year, } \\
\text { comorbidity and female gender. }\end{array}$ \\
\hline & & & & & & & $\begin{array}{l}\text { - For MCS: comorbidity and } \\
\text { purging behaviors }\end{array}$ \\
\hline \multirow{3}{*}{$\begin{array}{l}\text { [7] De La Rie } \\
\text { et al., } 2005\end{array}$} & \multirow{3}{*}{$\begin{array}{l}\text { Cross-sectional } \\
\text { study }\end{array}$} & \multirow{3}{*}{$\begin{array}{l}\text { Mixed: population via } \\
\text { advertisements and } \\
\text { ED centers }\end{array}$} & \multirow{3}{*}{$\begin{array}{l}156 \text { ED pts (44 AN, } 43 \\
\text { BN, } 69 \text { EDNOS) and } \\
148 \text { former ED pts }\end{array}$} & \multirow{3}{*}{$\begin{array}{l}\text { Dutch normative } \\
\text { population and } 591 \\
\text { Mood Disorders (MD) pts }\end{array}$} & \multirow{3}{*}{$\begin{array}{c}\text { DSM-IV diagnosis } \\
\text { based on EDE-Q + } \\
\text { BMI and menstrual } \\
\text { status }\end{array}$} & \multirow[t]{3}{*}{ SF-36 } & - No diff among ED groups. \\
\hline & & & & & & & • $\mathrm{ED}<$ normative. \\
\hline & & & & & & & - Former $\mathrm{ED}<$ normative. $\mathrm{ED}<\mathrm{MD}$ \\
\hline \multirow{3}{*}{$\begin{array}{l}\text { [11] Mond } \\
\text { et al., } 2005\end{array}$} & \multirow{3}{*}{$\begin{array}{l}\text { Cross-sectional } \\
\text { study }\end{array}$} & \multirow{3}{*}{$\begin{array}{l}\text { Pts referred to ED } \\
\text { treatment program }\end{array}$} & \multirow{3}{*}{$\begin{array}{l}87 \text { ED pts (34 AN, } \\
40 \mathrm{BN}, 10 \text { EDNOS) }\end{array}$} & \multirow{3}{*}{$\begin{array}{l}495 \text { general population } \\
\text { women }\end{array}$} & \multirow{3}{*}{$\begin{array}{c}\text { Clinical } \\
\text { assessment + EDE-Q }\end{array}$} & \multirow{3}{*}{$\begin{array}{l}\text { WHOQOL- } \\
\text { BREF }\end{array}$} & - ED pts < normative subjects. \\
\hline & & & & & & & $\begin{array}{l}\text { - Restricting AN pts > other } \\
\text { patient groups. }\end{array}$ \\
\hline & & & & & & & - BED $<$ other patients on PCS \\
\hline \multirow[t]{2}{*}{$\begin{array}{l}\text { [26] Engel } \\
\text { et al., } 2006\end{array}$} & \multirow[t]{2}{*}{$\begin{array}{l}\text { Cross-sectional } \\
\text { study to validate } \\
\text { EDQOL }\end{array}$} & \multirow[t]{2}{*}{$\begin{array}{l}538 \text { recruited sample } \\
\text { of student }\end{array}$} & \multirow[t]{2}{*}{155 ED, 56 diet/exercise } & \multirow[t]{2}{*}{$\begin{array}{l}\text { Validation across groups, } \\
\text { including } 327 \text { non-ED subj }\end{array}$} & \multirow[t]{2}{*}{$\begin{array}{l}\mathrm{SCID}+\mathrm{EDE}+ \\
\quad \mathrm{EAT}-26\end{array}$} & \multirow[t]{2}{*}{$\begin{array}{l}\text { EDQOL, } \\
\text { SF-36 }\end{array}$} & $\begin{array}{l}\text { - All EDQOL subscale scores } \\
\text { differed between groups, with } \\
\text { greater impairment in ED pts. }\end{array}$ \\
\hline & & & & & & & $\begin{array}{l}\text { - EDQOL more sensitive than } \\
\text { SF-36 when predicting group } \\
\text { status (ED vs. diet/exercise) }\end{array}$ \\
\hline \multirow[t]{2}{*}{$\begin{array}{l}\text { [27] De La Rie } \\
\text { et al., } 2007\end{array}$} & \multirow[t]{2}{*}{$\begin{array}{l}\text { Cross-sectional } \\
\text { study }\end{array}$} & \multirow{2}{*}{$\begin{array}{l}\text { Mixed: population via } \\
\text { advertisements and } \\
\text { ED centers }\end{array}$} & \multirow[t]{2}{*}{$\begin{array}{l}146 \text { ED pts (44 AN, } \\
43 \text { BN, } 59 \text { EDNOS) }\end{array}$} & \multirow[t]{2}{*}{146 former ED } & \multirow{2}{*}{$\begin{array}{c}\text { DSM-IV diagnosis } \\
\text { based on EDE-Q + } \\
\text { BMI and menstrual } \\
\text { status }\end{array}$} & SEIQOL & $\begin{array}{l}\text { - ED with poor QoL on all } \\
\text { life domains. }\end{array}$ \\
\hline & & & & & & & $\begin{array}{l}\text { - Former ED pts > ED pts on } \\
\text { most domains (but ratings } \\
\text { just above average) }\end{array}$ \\
\hline [28] Latner & Cross-sectional & ED outpatient center & $11 \mathrm{AN}, 5 \mathrm{BN}, 3 \mathrm{BED}$ & New Zealand normative & EDE-Q & SF-36 & ED < normative on MCS. \\
\hline & & & & & & & $\begin{array}{l}\text { - QoL general and PCS predicted } \\
\text { by subjective bulimic episodes }\end{array}$ \\
\hline $\begin{array}{l}\text { [29] Bamford \& } \\
\text { Sly, } 2010\end{array}$ & $\begin{array}{l}\text { Cross-sectional } \\
\text { study }\end{array}$ & ED outpatient center & $\begin{array}{l}156 \text { ED (80 AN, } \\
40 \text { BN, } 36 \text { EDNOS) }\end{array}$ & $\begin{array}{l}\text { Comparison across } \\
\text { ED groups }\end{array}$ & EDE-Q & EDQOL & $\begin{array}{l}\text { - AN }<\text { BN and EDNOS on } \\
\text { psychological and physical/ } \\
\text { cognitive domains }\end{array}$ \\
\hline $\begin{array}{l}\text { Baiano et al., } \\
\text { present study }\end{array}$ & $\begin{array}{l}\text { Cross-sectional } \\
\text { study }\end{array}$ & $\begin{array}{l}\text { ED center (in- and } \\
\text { out-patients) }\end{array}$ & $\begin{array}{l}80 \mathrm{ED} \text { (26 BN; } 33 \mathrm{AN} ; \\
7 \text { BED; } 14 \text { EDNOS) }\end{array}$ & $\begin{array}{l}\text { Comparison across } \\
\text { ED groups }\end{array}$ & $\begin{array}{l}\text { Clinical interview } \\
\text { (DSM-IV) }\end{array}$ & $\begin{array}{l}\text { WHOQOL- } \\
\text { BREF }\end{array}$ & $\begin{array}{l}\text { - No diff among ED groups. } \\
\text { EDNOS > other groups on } \\
\text { psychological health QoL }\end{array}$ \\
\hline Obese patients & & & & & & & \\
\hline $\begin{array}{l}\text { [30] Hsu } \\
\text { et al., } 2002\end{array}$ & $\begin{array}{l}\text { Cross-sectional } \\
\text { study }\end{array}$ & 37 subj awaiting GBP & 9 BED & 28 non-BED & EDE, TFEQ, SCID-IV & SF-36 & $\cdot$ BED $<$ non-BED \\
\hline $\begin{array}{l}\text { [31] De Zwaan } \\
\text { et al., } 2002\end{array}$ & $\begin{array}{l}\text { Cross-sectional study } \\
\text { on pre and }\end{array}$ & 78 obese surgical pts & $\begin{array}{l}78 \text { obese ( } 9 \text { BED) } \\
\text { after GBP surgery }\end{array}$ & $\begin{array}{l}110 \text { preoperative } \\
\text { control group (19 BED) }\end{array}$ & $\begin{array}{l}\text { Phone interview + } \\
\text { MFED + QWEPR }\end{array}$ & SF-36 & $\begin{array}{l}\text { - Postoperative pts > } \\
\text { preoperative pts. }\end{array}$ \\
\hline & $\begin{array}{l}\text { post-operative } \\
\text { patients }\end{array}$ & & & & & & $\begin{array}{l}\text { - Postoperative pts }<\text { US } \\
\text { norm values on PCS }\end{array}$ \\
\hline [32] Masheb \& & Cross-sectional & Pts undergoing a medical & 94 BED & US normative population & Clinical interview & SF-36 & - BED < normative. \\
\hline & & & & binges $(n=312)$ & & & - BED $<$ non-BED on PCS \\
\hline
\end{tabular}


Table 1 Review of the literature on QoL in eating disorders subjects (Continued)

\begin{tabular}{|c|c|c|c|c|c|c|c|}
\hline $\begin{array}{l}\text { [33] Kolotkin } \\
\text { et al., } 2004\end{array}$ & $\begin{array}{l}\text { Cross-sectional } \\
\text { study }\end{array}$ & $\begin{array}{l}530 \text { obese candidates to } \\
\text { residential modification } \\
\text { program }\end{array}$ & $95 \mathrm{BED}$ & 435 non-BED & $\begin{array}{c}\text { Questionnaire on } \\
\text { Eating/Weight Patterns; BDI; } \\
\text { SC90-R }\end{array}$ & IWQOL-Lite & $\begin{array}{l}\text { - } \mathrm{BED}=\text { non-BED when other } \\
\text { variables are considered }\end{array}$ \\
\hline $\begin{array}{l}\text { [34] Rieger } \\
\text { et al., } 2005\end{array}$ & $\begin{array}{l}\text { Cross-sectional study } \\
\text { within a RCT }\end{array}$ & $\begin{array}{l}118 \text { treatment-seeking } \\
\text { obese subj }\end{array}$ & $56 \mathrm{BED}$ & 62 non-BED & EDE, PRIME-MD & IWQOL-Lite & $\begin{array}{l}\text { - BED }<\text { non-BED on total scale, } \\
\text { but not on physical function } \\
\text { subscale }\end{array}$ \\
\hline $\begin{array}{l}\text { [35] Colles } \\
\text { et al., } 2008\end{array}$ & Cross-sectional study & $\begin{array}{l}180 \text { bariatric surgery } \\
\text { candidates, } 93 \text { participants } \\
\text { to a weight loss support } \\
\text { group, } 158 \text { community } \\
\text { respondents }\end{array}$ & $\begin{array}{l}38 \text { BED, } 46 \text { subj with } \\
\text { feelings of loss of } \\
\text { control (LOC) during } \\
\text { binge episodes }\end{array}$ & 307 non-binge eaters & $\begin{array}{c}\text { QEWP-R }+ \\
\text { semistructured } \\
\text { interview or phone } \\
\text { interview }\end{array}$ & SF-36 & - BED $<$ non-BED on MCS \\
\hline $\begin{array}{l}\text { [36] Folope } \\
\text { et al., } 2012\end{array}$ & Cross-sectional study & $\begin{array}{l}130 \text { obese in clinical } \\
\text { nutrition center }\end{array}$ & $73 \mathrm{ED}$ & 57 non-ED & $\begin{array}{l}\text { SCOFF-F + BULIT, } \\
\text { not confirmed by } \\
\text { a diagnosis }\end{array}$ & QOLOD & $\begin{array}{l}\cdot E D<\text { non-ED, globally and on } \\
\text { psychological dimension }\end{array}$ \\
\hline \multirow{2}{*}{$\begin{array}{l}\text { [37] Ranzenhofer } \\
\text { et al., } 2012\end{array}$} & \multirow[t]{2}{*}{ Cross-sectional study } & \multirow{2}{*}{$\begin{array}{l}158 \text { obese adolescents } \\
\text { selected for weight-loss } \\
\text { treatment }\end{array}$} & \multirow{2}{*}{$\begin{array}{l}35 \text { binge eating } \\
(6 \text { proper } \mathrm{BED})\end{array}$} & \multirow[t]{2}{*}{123 non-binge eaters } & \multirow[t]{2}{*}{ EDE } & \multirow[t]{2}{*}{ IWQOL-A } & • Binge eating < no-binge eating. \\
\hline & & & & & & & $\begin{array}{l}\text { - Girls with binge eating < boys } \\
\text { with binge eating }\end{array}$ \\
\hline \multicolumn{8}{|l|}{ Cohort studies } \\
\hline \multicolumn{8}{|l|}{ Survey } \\
\hline $\begin{array}{l}\text { [38] Wade } \\
\text { et al., } 2012\end{array}$ & $\begin{array}{l}\text { Longitudinal multi- } \\
\text { wave survey }\end{array}$ & $\begin{array}{l}\text { 9,688 population of } \\
\text { women }\end{array}$ & 2223 ED & 7465 non-ED & $\begin{array}{l}\text { Ad-hoc questionnaire, } \\
\text { EDE-Q, not confirmed } \\
\text { by interview }\end{array}$ & SF-36 & $\begin{array}{l}\cdot E D<\text { non-ED, globally and on } \\
P C S \text { and MCS }\end{array}$ \\
\hline \multicolumn{8}{|c|}{ ED outpatient treatments } \\
\hline \multirow[t]{3}{*}{$\begin{array}{l}\text { [39] Padierna } \\
\text { et al., } 2002\end{array}$} & \multirow[t]{3}{*}{2 years cohort study } & \multirow[t]{3}{*}{ ED outpatient center } & \multirow[t]{3}{*}{131 ED (90 AN, 41 BN) } & \multirow[t]{3}{*}{$\begin{array}{l}\text { Spanish normative } \\
\text { population }\end{array}$} & \multirow[t]{3}{*}{$\begin{array}{l}\text { Clinical interview } \\
\text { (DSM-IV) }\end{array}$} & \multirow[t]{3}{*}{ SF-36 } & $\begin{array}{l}\text { - Improvement in PCS and social } \\
\text { function, followed by MCS. }\end{array}$ \\
\hline & & & & & & & $\begin{array}{l}\text { - Scores after } 2 \text { years still below } \\
\text { normative population. }\end{array}$ \\
\hline & & & & & & & $\begin{array}{l}\text { - Severity of ED affected } \\
\text { improvement }\end{array}$ \\
\hline \multirow{3}{*}{$\begin{array}{l}\text { [40] Muñoz } \\
\text { et al., } 2009\end{array}$} & \multirow{3}{*}{$\begin{array}{c}\text { Cohort study (baseline, } \\
\text { after } 1 \text { year) }\end{array}$} & \multirow{3}{*}{$\begin{array}{l}358 \text { subj in treatment } \\
\text { programs in Health centers }\end{array}$} & \multirow{3}{*}{$\begin{array}{l}61 \text { AN, } 47 \text { BN, } \\
245 \text { EDNOS }\end{array}$} & \multirow{3}{*}{$\begin{array}{c}305 \text { general population } \\
\text { women }\end{array}$} & \multirow{3}{*}{$\begin{array}{l}\text { Clinical interview } \\
\text { (DSM-IV) }\end{array}$} & \multirow{3}{*}{$\begin{array}{l}\text { HeRQoLED, } \\
\text { SF-36 }\end{array}$} & - ED < general population. \\
\hline & & & & & & & $\begin{array}{l}\text { - After } 1 \text { year PCS improved } \\
\text { but not MCS. }\end{array}$ \\
\hline & & & & & & & $\begin{array}{l}\text { - AN < other ED at baseline, } \\
\text { and smaller improvements } \\
\text { after } 1 \text { year }\end{array}$ \\
\hline \multirow[t]{2}{*}{$\begin{array}{l}\text { [41] Adair } \\
\text { et al., } 2010\end{array}$} & \multirow{2}{*}{$\begin{array}{c}\text { Cohort study (baseline, } \\
3 \text { and } 6 \text { months } \\
\text { follow-ups) to validate } \\
\text { EDQLS }\end{array}$} & \multirow[t]{2}{*}{ ED treatment programs } & \multirow[t]{2}{*}{$\begin{array}{l}130 \mathrm{ED} \text { pts (56 AN, } \\
39 \mathrm{BN}, 35 \text { EDNOS) }\end{array}$} & \multirow[t]{2}{*}{$\begin{array}{l}\text { QoL measures at } \\
\text { different point in time }\end{array}$} & \multirow[t]{2}{*}{ Clinical } & \multirow{2}{*}{$\begin{array}{l}\text { EDQLS, } \\
\text { Quality of } \\
\text { Life } \\
\text { Inventory, } \\
\text { SF-12 }\end{array}$} & $\begin{array}{l}\text { - EDQLS total scores increased } \\
\text { at } 3 \text { and } 6 \text { months. }\end{array}$ \\
\hline & & & & & & & $\begin{array}{l}\text { - EDQLS responsiveness exceeded } \\
\text { that of other QoL instruments }\end{array}$ \\
\hline
\end{tabular}


Table 1 Review of the literature on QoL in eating disorders subjects (Continued)

\section{Specific}

\section{treatments}

42] Marchesin

et al., 2002

ntervention study

(3-5 months CBT)

96 obese enrolled in a

CBT program

46 BED

76 untreated controls

[43] Nickel

Intervention study

60 BN women recruited

through advertisements topiramate)

[44] Wiffley

et al., 2008

Intervention study

(24 weeks RCT with sibutramine)

Residential

\section{treatments}

[45] Abraham

et al., 2006

Cohort study (baseline, at discharge and

12 months follow-up)

[46] McHugh, Prospective residentia

2007

$$
\text { cohort study }
$$

ED residential center

65 AN (33 high

Readiness for

Change - RFC - females

vs. 32 low RFC females)

Comparison between

high- and low-RFC

\section{Surgical}

interventions

[47] Green

et al., 2004
65 surgical (GBP) pts

33 BED

32 non-BED
Clinical interview (DSM-IV)

Interview, BES, EDE

SCID-I and SCD-II

EDE

Clinical interview

EEE-C

QOLscores,

SF12

- QoL improved during inpatient treatment and between admission and 12 months after discharge.

- AN, BN and EDNOS < no diagnosis.

- Specific differences among ED groups on some dimensions

SF-36 V2 - Participants' QoL below US average. - $81 \%$ discharged below the US average.

- No diff between RFC and non-RFC

ED-SCID, QWEP-R
Glossary of QoL instruments.

$A Q O L$, assessment of quality of

$E D E-Q$, eating disorder examination questionnaire.

$E D Q L S$, eating disorders quality of life.

$E E E-C Q O L$, eating and exercise examination QOL.

HeRQOLED, health related quality of life for the eating disorders.

HWRQOLED, health related quality of life for the
IWQOLA

Kindl-Re Revised Geight on quality of life-lite.

NHP, Noting Ge

$Q O L O D$, quality of life, obesity and dietetics rating scale.

SEIQOL, schedule for the evaluation of individual quality of life.

SF-36, short form (36) health survey.

WHOQOL-Bref: brief version of the world health organization quality of life questionnaire
- Ool improved from pre-surgery to post-surgery. - BED $<$ non-BED on social functioning at pre-surgery and after 6 months postsurgery 
Table 2 Socio-demographic and clinical characteristics of ED patients: continuous variables

\begin{tabular}{lcccccc}
\hline & AN $(\mathbf{N}=\mathbf{3 3})$ & BN $(\mathbf{N}=\mathbf{2 6})$ & BED $(\mathbf{N}=\mathbf{7})$ & EDNOS $(\mathbf{N}=14)$ & Kruskal-Wallis test & Significance \\
\hline Age (years) & $26.09 \pm 10.59$ & $27.46 \pm 7.96$ & $41.57 \pm 14.01$ & $28.07 \pm 13.28$ & $X^{2}=8.293$ & $p=0.040$ \\
BMI $\left(\mathbf{k g} / \mathbf{m}^{2}\right)$ & $15.97 \pm 1.88$ & $21.99 \pm 5.17$ & $32.40 \pm 8.26$ & $24.73 \pm 7.29$ & $X^{2}=54.916$ & $p<0.001$ \\
Age at onset (years) & $19.21 \pm 6.93$ & $17.92 \pm 5.37$ & $36.14 \pm 15.40$ & $22.57 \pm 13.20$ & $X^{2}=9.585$ & $p=0.022$ \\
Age at first evaluation (years) & $26.06 \pm 10.61$ & $27.39 \pm 8.00$ & $41.57 \pm 14.01$ & $28.07 \pm 13.28$ & $X^{2}=8.198$ & $p=0.040$ \\
Lenght of illness (years) & $6.88 \pm 7.27$ & $9.54 \pm 7.20$ & $5.43 \pm 7.00$ & $5.50 \pm 9.97$ & $X^{2}=9.456$ & $p=0.020$ \\
\hline
\end{tabular}

Mean \pm SD.

HRQoL scores were not significantly correlated to length of illness within each ED subgroup ( $p>0.05$, Pearson's correlation analysis).

\section{Discussion}

The main evidences coming out from our review are the followings:
- the surveys confirmed that ED patients have a poorer HRQoL than the general population and this is evident in particular when considering the mental component of the quality of life;

- among the obese patients, quite invariably the BED had a poorer HRQoL than non-BED subjects. This is an important piece of information, since it

Table 3 Socio-demographic and clinical characteristics of ED patients: categorical variables

\begin{tabular}{|c|c|c|c|c|c|}
\hline & AN $(\mathrm{N}=33)$ & $\mathrm{BN}(\mathrm{N}=26)$ & BED $(N=7)$ & EDNOS $(\mathrm{N}=14)$ & Fisher's exact test \\
\hline Occupation & & & & & $P=0.070$ \\
\hline Yes & $7(21.2 \%)$ & $13(50 \%)$ & $4(57.1 \%)$ & $7(50 \%)$ & \\
\hline No & $26(78.8 \%)$ & $13(50 \%)$ & $3(42.9 \%)$ & $7(50 \%)$ & \\
\hline Marital status & & & & & $P<0.001$ \\
\hline Unmarried & 29 (87.9\%) & $21(80.7 \%)$ & $1(14.3 \%)$ & $11(78.6 \%)$ & \\
\hline Married or coupled & $3(9.1 \%)$ & $2(7.7 \%)$ & 5 (71.4\%) & $3(21.4 \%)$ & \\
\hline Divorced or separated & $1(3.0 \%)$ & $3(11.5 \%)$ & $1(14.3 \%)$ & 0 & \\
\hline Educational level & & & & & $P=0.093$ \\
\hline Primary school & $16(48.5 \%)$ & $5(19.2 \%)$ & $5(71.4 \%)$ & $6(42.9 \%)$ & \\
\hline High school & $11(33.3 \%)$ & $17(65.4 \%)$ & $2(28.6 \%)$ & $5(35.7 \%)$ & \\
\hline University & $6(18.2 \%)$ & $4(15.4 \%)$ & 0 & $3(21.4 \%)$ & \\
\hline Living conditions & & & & & $P=1.000$ \\
\hline Alone & $3(9.1 \%)$ & $2(7.7 \%)$ & 0 & $1(7.1 \%)$ & \\
\hline With others & $30(90.9 \%)$ & $24(92.3)$ & $7(100 \%)$ & $13(92.9 \%)$ & \\
\hline Patients completing education & & & & & $P=0.810$ \\
\hline Yes & $14(42.4 \%)$ & $8(30.8 \%)$ & $2(28.6 \%)$ & $5(35.7 \%)$ & \\
\hline No & $19(57.6 \%)$ & $18(69.2 \%)$ & $5(71.4 \%)$ & $9(64.3 \%)$ & \\
\hline Setting & & & & & $P=0.005$ \\
\hline Inpatients & $14(42.4 \%)$ & 7 (26.9\%) & 0 & 0 & \\
\hline Outpatients & $19(57.6 \%)$ & $19(73.1 \%)$ & $7(100 \%)$ & $14(100 \%)$ & \\
\hline Previous treatment for ED & & & & & $P<0.001$ \\
\hline Yes & $27(81.8 \%)$ & $14(53.8 \%)$ & $1(14.3 \%)$ & $4(28.6 \%)$ & \\
\hline No & $6(18.2 \%)$ & $12(46.1 \%)$ & $6(85.7 \%)$ & $10(71.4 \%)$ & \\
\hline DSM-IV comorbidity & & & & & $P=0.431$ \\
\hline Yes & $7(21.2 \%)$ & $6(23.1 \%)$ & 0 & 0 & \\
\hline No & $26(78.8 \%)$ & $20(76.9 \%)$ & $7(100 \%)$ & $14(100 \%)$ & \\
\hline Illness' awareness & & & & & $P=0.548$ \\
\hline Yes & $21(63.6 \%)$ & $16(61.5 \%)$ & $5(71.4 \%)$ & $6(42.9 \%)$ & \\
\hline No & $12(36.4 \%)$ & $10(38.5 \%)$ & $2(28.6 \%)$ & $8(57.1 \%)$ & \\
\hline
\end{tabular}


Table 4 HRQoL scores among ED subgroups

\begin{tabular}{|c|c|c|c|c|}
\hline HRQoL domains & ED diagnosis & Mean SD & Statistics & Significance \\
\hline \multirow{4}{*}{ Physical } & AN & $12.69 \pm 3.09$ & \multirow{4}{*}{$F=0.638$} & \multirow{4}{*}{$p=0.593$} \\
\hline & $B N$ & $12.39 \pm 3.40$ & & \\
\hline & $B E D$ & $11.75 \pm 2.21$ & & \\
\hline & EDNOS & $13.59 \pm 3.55$ & & \\
\hline \multirow{4}{*}{ Psychological } & AN & $11.28 \pm 1.28$ & \multirow{4}{*}{$F=4.390$} & \multirow{4}{*}{$p=0.007 \wedge$} \\
\hline & $B N$ & $11.04 \pm 1.45$ & & \\
\hline & $B E D$ & $10.52 \pm 0.94$ & & \\
\hline & EDNOS & $12.56 \pm 1.96$ & & \\
\hline \multirow{4}{*}{ Social } & AN & $10.87 \pm 3.86$ & \multirow{4}{*}{$F=0.772$} & \multirow{4}{*}{$p=0.513$} \\
\hline & $B N$ & $10.76 \pm 3.01$ & & \\
\hline & $B E D$ & $11.43 \pm 4.21$ & & \\
\hline & EDNOS & $12.47 \pm 4.10$ & & \\
\hline \multirow{4}{*}{ Environment } & AN & $12.35 \pm 2.55$ & \multirow{4}{*}{$F=0.383$} & \multirow{4}{*}{$p=0.766$} \\
\hline & $B N$ & $12.80 \pm 2.09$ & & \\
\hline & $B E D$ & $13.14 \pm 3.36$ & & \\
\hline & EDNOS & $12.96 \pm 2.02$ & & \\
\hline
\end{tabular}

supports the idea that there is a need of specific treatments for BED, which are not simply an adaptation of general nutrition intervention;

- in the cohort studies, we can distinguish general programs aimed to treat the ED from specific psychotherapies and RCT with psychotropic drugs. Even if the published data seem to reassure that many treatments are able to improve the HRQoL, we remain with the doubt whether the amelioration was due to some specific characteristics of the treatment or was a generic result of the caring of these patients. Much has to be done in order to respond to the question "what aspect of treatment improve HRQoL of ED patients?”.

Furthermore, we must highlight the fact that most studies used general scales for measuring the HRQoL, and in particular the SF-36. Since much fewer information comes from studies that used specific scales (such as EDQOL), so far it is not possible to draw conclusion on the advantages of the latter scales. On the other hand, the analysis of Table 1 may suggest that the use of ED specific scales is able to increase the probability to discover subtle differences in HRQoL among different ED groups or between pre- and posttreatment scores.

When we consider our cross-sectional study, we must admit that our sample is of relatively small size, even if it is on average with other studies. To overcome this limit we used a non-parametric test (Kruskall-Wallis test), which in principle does not put limits in the sample size.
The main difference of our research as compared with other studies is that we included both inpatients and outpatients. It is worth noting that over $40 \%$ of outpatients, but none of the inpatients, had a history of previous treatments. If we assume that inpatients have a more severe illness, we may speculate that these patients did not seek help because of their low illness' awareness and, in addition, that primary care physicians did not identify an ED in these subjects, although they were presenting important weight alterations, repetitive vomiting or bingeing [48]. Therefore, specific interventions were dramatically delayed until the need for an admission was necessary.

Additionally, over $85 \%$ of patients with comorbid psychiatric diagnoses reported a history of previous treatment for ED, in comparison to $50 \%$ of patients without coexisting psychiatric diagnoses. These results may indicate that the presence of comorbidity increased the probability to be treated earlier. We know from previous surveys that ED are frequently comorbid with Major Depressive Disorder, Anxiety Disorders and Substance or Alcohol Abuse [49] and also that ED relapses and successive hospitalizations reduce the response to treatment of comorbidity [50,51]. We may conclude that the comorbidity of severe ED psychopathology with other psychiatric disorders contributed to chronicity of illness and should be considered as a prognostic factor influencing long-term outcomes of rehabilitation programs and increasing financial dependence on society [52-54].

As far as HRQoL is concerned, our study confirmed results obtained by previous investigations, demonstrating that ED patients experienced reduced HRQoL. Surprisingly, 
ED inpatients and outpatients did not differ as for HRQoL scores. Most ED inpatients in our sample were AN, thus confirming that anorexic behaviors implicate a high risk of organic complications and require a large amount of intensive medical care [55]. It is possible that AN inpatients did not report greater limitations of QoL in comparison to the other subgroups, because of a poor perception of psychosomatic distress [56,57]. Moreover, we found no differences in HRQoL between ED patients with and without a comorbid DSM-IV diagnosis, as well as between patients with and without a history of previous treatments. Furthermore, HRQoL scores were not significantly correlated to length of illness within each ED subgroup. Taken together, all these negative results may indicate that our patients were impaired in their quality of life independently from their history and current severity of illness.

\section{Conclusions}

Finally, when we consider the specific domains of HRQoL involved, the only difference among the ED groups was present in the Psychological component, where patients with EDNOS reported the highest HRQoL scores and patients with BED the lowest ones. In fact EDNOS patients represent a heterogeneous category of subjects with eating behaviors generating a relatively modest distress [58,59], and our EDNOS patients seem to confirm this rule. Also the data on our patients with BED are in agreement with previous studies, which documented great health dissatisfaction, increased risk of main medical disorders [60], difficulties in regulating emotions [61] and concurrence of personality traits, as well as comorbidity with mood and anxiety disorders $[62,63]$ in binge eaters. All together, these last results may indicate the need in the future studies to focus the attention in particular on the characteristics of the psychological domain of HRQoL in eating disorders.

\section{Competing interests}

In the past five years none of the Authors received reimbursements, fees, funding, or salary from an organization that may in any way gain or lose financially from the publication of this manuscript, either now or in the future. None of the Authors hold any stocks or shares in an organization that may in any way gain or lose financially from the publication of this manuscript, either now or in the future. There are not non-financial competing interests (political, personal, religious, ideological, academic, intellectual, commercial or any other) to declare in relation to this manuscript.

\section{Authors' contributions}

MoB and PS are the principal investigators of the cross-sectional study and MoB performed the statistical analysis of the study. PR, LC, EB and IC participated in the design of the cross-sectional study and collected the data. They were involved in the critical revision of the manuscript. MaB participated in the design of the cross-sectional study and carried out the review of the literature. He also drafted the manuscript, and takes the main responsibility for the interpretation of data. All authors read and approved the final manuscript.

\section{Acknowledgements}

We wish to thank the Socio-Health Unit (ASSL "Veneto Orientale", Portogruaro, Italy) for the possibility to use the clinical data for this research.

\section{Author details}

${ }^{1}$ Centre for Weight and Eating Disorders, Azienda Socio-Sanitaria Locale n. 10 "Veneto Orientale", Venice, Portogruaro, Italy. ${ }^{2}$ Unit of Psychiatry, Dept. of Experimental and Clinical Medical Sciences, University of Udine, Udine, Italy. ${ }^{3}$ Clinica Psichiatrica, Azienda Ospedaliero-Universitaria, P.le S.M. Misericordia 15, 34070 Udine, Italy.

Received: 16 May 2013 Accepted: 19 February 2014

Published: 4 June 2014

\section{References}

1. The WHOQOL Group: Development of the World Health Organization WHOQOL-BREF Quality of Life assessment. Psychol Med 1998, 28:551-558.

2. Centers for Disease Control and Prevention: Measuring healthy days: Population assessment of health-related quality of life. Atlanta, Georgia: CDCP; 2000.

3. Engel SG, Adair CE, Las Hayas C, Abraham S: Health related quality of life and eating disorders: a review and an update. Int J Eat Disord 2009, 42:179-187.

4. Boini S, Briançon S, Guillemin F, Galan P, Hercberg S: Impact of cancer occurrence on health-related quality of life: a longitudinal pre-post assessment. Health Qual Life Outcomes 2004, 2:4.

5. Bullinger M: Health-related quality of life and subjective health. Psychother Psychosom Med Psychol 1997, 47:76-91.

6. Guyatt GH, Feeny D, Patrick DL: Measuring health-related quality of life. Ann Intern Med 1993, 118:622-629.

7. De la Rie S, Noordenbos G, Donker M, van Furth E: Quality of life and eating disorders. Qual Life Res 2005, 14:1511-1522.

8. Bosanac P, Kurlender S, Stojanovska L, Hallam K, Norman T, McGrath C, Burrows G, Wesnes K, Manktelow T, Olver J: Neuropsychological study of underweight and "weight-recovered" anorexia nervosa compared with bulimia nervosa and normal controls. Int J Eat Disord 2007, 40:613-621.

9. Fowler L, Blackwell A, Jaffa A, Palmer R, Robbins TW, Sahakian BJ, Dowson $\mathrm{JH}$ : Profile of neurocognitive impairments associated with female in-patients with anorexia nervosa. Psychol Med 2006, 36:517-527.

10. Padierna A, Quintana JM, Arostegui I, Gonzalez N, Horcajo MJ: The health-related quality of life in eating disorders. Qual Life Res 2000, 9:667-674.

11. Mond JM, Hay PJ, Rodgers B, Owen C, Beumont PJV: Assessing quality of life in eating disorder patients. Qual Life Res 2005, 14:171-178.

12. Davies M, Brody D, deGruy FV 3rd, Spitzer RL, Kroenke K, Linzer M, Hahn SR, Williams JB: Health-related quality of life in primary care patients with mental disorders. Results from the PRIME-MD 1000 Study. JAMA 1995, 274:1511-1517.

13. Bohn K, Doll HA, Cooper Z, O'Connor M, Palmer RL, Fairburn CG: The measurement of impairment due to eating psychopathology. Behav Res Ther 2008, 46:1105-1110.

14. Passarelli Tirico P, Stefano SC, Blay SL: Quality of life and eating disorders: a systematic review. Cad Saude Publica 2010, 26:431-449.

15. Jenkins PE, Hoste RR, Meyer C, Blissett JM: Eating disorders and quality of life: a review of the literature. Clin Psychol Rev 2011, 31:113-121.

16. SPSS Inc: SPSS for Windows, Version 16.0. Chicago: SPSS Inc; 2007.

17. StataCorp: Stata Statistical Software: Release 10. College Station, TX: StataCorp LP; 2007.

18. Hay P: Quality of life and bulimic eating disorder behaviours: findings from a community-based sample. Int J Eat Disord 2003, 33:434-442.

19. Doll HA, Petersen SE, Stewart-Brown SL: Eating disorders and emotional and physical well-being: associations between student self-reports of eating disorders and quality of life as measured by the SF-36. Qual Life Res 2005, 14:705-717.

20. Herpetz-Dahlmann B, Wille N, Hölling H, Vloet TD, Ravens-Sieberer U, BELLA study group: Disordered eating behaviour and attitudes, associated psychopathology and health-related quality of life: results of the BELLA study. Eur Child Adolesc Psychiatry 2008, 17:82-91.

21. Vallance JK, Latner JD, Gleaves DH: The relationship between eating disorder psychopathology and health-related quality of life within a community sample. Qual Life Res 2011, 20:675-682. 
22. Mond J, Hay P, Rodgers B, Owen C: Quality of life impairment in a community sample of women with eating disorders. Aust N Z J Psychiatry 2012, 46:561-568.

23. Mitchison D, Hay P, Mond J, Slewa-Younan S: Self-reported history of anorexia nervosa and current quality of life: findings from a community-based study. Qual Life Res 2013, 22:273-281.

24. Keilen M, Treasure T, Schmidt U, Treasure J: Quality of life measurements in eating disorders, angina, and transplant candidates: are they comparable? J R Soc Med 1994, 87:441-444.

25. González-Pinto A, Inmaculada F, Cristina R, de Corres BF, Sonsoles E, Fernando R, Purificacion L: Purging behaviors and comorbidity as predictive factors of quality of life in anorexia nervosa. Int J Eat Disord 2004, 36:445-450.

26. Engel SG, Wittrock DA, Crosby RD, Wonderlich SA, Mitchell JE, Kolotkin RL: Development and psychometric validation of an eating disorder-specific health-related quality of life instrument. Int J Eat Disord 2006, 39:62-71.

27. De la Rie S, Noordenbos G, Donker M, van Furth $E$ : The patient's view on quality of life and eating disorders. Int J Eat Disord 2007, 40:13-20.

28. Latner JD, Vallance JK, Buckett G: Health-related quality of life in women with eating disorders: association with subjective and objective binge eating. J Clin Psychol Med Settings 2008, 15:148-153.

29. Bamford B, Sly R: Exploring quality of life in the eating disorders. Eur Eat Disord Rev 2010, 18:147-153.

30. Hsu LK, Mulliken B, McDonagh B, Krupa Das S, Rand W, Fairburn CG, Rolls B, McCrory MA, Saltzman E, Shikora S, Dwyer J, Roberts S: Binge eating disorder in extreme obesity. Int J Obes Relat Metab Disord 2002, 26:1398-1403.

31. de Zwaan M, Lancaster KL, Mitchell JE, Howell LM, Monson N, Roerig JL, Crosby RD: Health-related quality of life in morbidly obese patients: effect of gastric bypass surgery. Obes Surg 2002, 12:773-780.

32. Masheb RM, Grilo CM: Quality of life in patients with binge eating disorder. Eat Weight Disord 2004, 9:194-199.

33. Kolotkin RL, Westman EC, Østbye T, Crosby RD, Eisenson HJ, Binks M Does binge eating disorder impact weight-related quality of life? Obes Res 2004, 12:999-1005.

34. Rieger E, Wilfley DE, Stein RI, Marino V, Crow SJ: A comparison of quality of life in obese individuals with and without binge eating disorder. Int J Eat Disord 2005, 37:234-240.

35. Colles SL, Dixon JB, O'Brien PE: Loss of control is central to psychological disturbance associated with binge eating disorder. Obesity (Silver Spring) 2008, 16:608-614

36. Folope V, Chapelle C, Grigioni S, Coëffier M, Déchelotte P: Impact of eating disorders and psychological distress on the quality of life of obese people. Nutrition 2012, 28:e7-e13.

37. Ranzenhofer LM, Columbo KM, Tanofsky-Kraff M, Shomaker LB, Cassidy O, Matheson BE, Kolotkin RL, Checchi JM, Keil M, McDuffie JR, Yanovski JA: Binge eating and weight-related quality of life in obese adolescents. Nutrients 2012, 4:167-180.

38. Wade TD, Wilksch SM, Lee C: A longitudinal investigation of the impact of disordered eating on young women's quality of life. Health Psychol 2012, 31:352-359.

39. Padierna A, Quintana JM, Arostegui I, Gonzalez N, Horcajo MJ: Changes in health related quality of life among patients treated for eating disorders. Qual Life Res 2002, 11:545-552.

40. Muñoz P, Quintana JM, Las Hayas C, Aguirre U, Padierna A, González-Torres $M A$ : Assessment of the impact of eating disorders on quality of life using the disease-specific, health-related quality of life for eating disorders (HeRQoLED) questionnaire. Qual Life Res 2009, 18:1137-1146.

41. Adair CE, Marcoux GC, Bischoff TF, Cram BS, Ewashen CJ, Pinzon J, Gusella J, Geller J, Scattolon Y, Fergusson P, Styles L, Brown KE: Responsiveness of the eating disorders quality of life scale (EDQLS) in a longitudinal multi-site sample. Health Qual Life Outcomes 2010, 8:83.

42. Marchesini G, Natale S, Chierici S, Manini R, Besteghi L, Di Domizio S, Sartini A, Pasqui F, Baraldi L, Forlani G, Melchionda N: Effects of cognitive-behavioural therapy on health-related quality of life in obese subjects with and without binge eating disorder. Int J Obes Relat Metab Disord 2002, 26:1261-1267.

43. Nickel C, Tritt K, Muehlbacher M, Pedrosa Gil F, Mitterlehner FO, Kaplan P, Lahmann C, Leiberich PK, Krawczyk J, Kettler C, Rother WK, Loew TH, Nickel MK: Topiramate treatment in bulimia nervosa patients: a randomized, double-blind, placebo-controlled trial. Int J Eat Disord 2005, 38:295-300.
44. Wilfley DE, Crow SJ, Hudson JI, Mitchell JE, Berkowitz RI, Blakesley V, Walsh BT, Sibutramine Binge Eating Disorder Research Group: Efficacy of sibutramine for the treatment of binge eating disorder: a randomized multicenter placebo-controlled double-blind study. Am J Psychiatry 2008, 165:51-58.

45. Abraham SF, Brown T, Boyd C, Luscombe G, Russel J: Quality of life: eating disorders. Aust New Zeal J Psychiatr 2006, 40:150-155.

46. McHugh MD: Readiness for change and short-term outcomes of female adolescents in residential treatment for anorexia nervosa. Int J Eat Disord 2007, 40:602-612.

47. Green AE, Dymek-Valentine M, Pytluk S, Le Grange D, Alverdy J: Psychosocial outcome of gastric bypass surgery for patients with and without binge eating. Obes Surg 2004, 14:975-985.

48. Karwautz A, de Zwaan M, Wöber-Bingöl C, Wöber C, Friedrich MH: Awareness of early-onset anorexia nervosa. Eat Weight Disord 1997, 2:138-143

49. Spindler A, Milos G: Links between eating disorder symptom severity and psychiatric comorbidity. Eat Behav 2007, 8:364-373.

50. Sihvola E, Keski-Rahkonen A, Dick DM, Hoek HW, Raevuori A, Rose RJ, Pulkkinen L, Marttunen M, Kaprio J: Prospective associations of early-onset Axis I disorders with developing eating disorders. Compr Psychiatry 2009, 50:20-25

51. Woodside BD, Staab R: Management of psychiatric comorbidity in anorexia nervosa and bulimia nervosa. CNS Drugs 2006, 20:665-663.

52. Blinder BJ, Cumella EJ, Sanathara VA: Psychiatric comorbidities of female inpatients with eating disorders. Psychosom Med 2006, 68:454-462.

53. Hjern A, Lindberg L, Lindblad F: Outcome and prognostic factors for adolescent female in-patients with anorexia nervosa: 9-to14-year follow-up. Br J Psychiatry 2006, 18:428-432.

54. Steinhausen HC: The outcome of anorexia nervosa in the 20th century. Am J Psychiatry 2002, 59:1284-1293.

55. Patrick L: Eating Disorders: a review of the literature with emphasis on medical complications and clinical nutrition. Altern Med Rev 2002, 7:184-202.

56. Gilboa-Schechtman E, Avnon L, Zubery E, Jeczmien P: Emotional processing in eating disorders: specific impairment or general distress related deficiency? Depress Anxiety 2006, 23:331-339.

57. Pollatos O, Kurz AL, Albrecht J, Schreder T, Kleemann AM, Schöpf V, Kopietz R, Wiesmann M, Schandry R: Reduced perception of bodily signals in anorexia nervosa. Eat Behav 2008, 9:381-388.

58. Hudson Jl, Hiripi E, Pope HG, Kessler RC: The prevalence and correlates of eating disorders in the National Comorbidity Survey Replication. Biol Psychiatry 2007, 61:348-358.

59. Reba-Harrelson L, Von Holle A, Hamer RM, Swann R, Reyes ML, Bulik CM: Patterns and prevalence of disordered eating and weight control behaviors in women ages 25-45. Eat Weight Disord 2009, 14:190-198.

60. Bulik CM, Sullivan PF, Kendler KS: Medical and psychiatric morbidity in obese women with and without binge eating. Int J Eat Disord 2002, 32:72-78.

61. Whiteside U, Chen E, Neighbors C, Hunter D, Lo T, Larimer M: Difficulties regulating emotions: do binge eaters have fewer strategies to modulate and tolerate negative affect? Eat Behav 2007, 8:162-169.

62. Peterson CB, Miller KB, Crow SJ, Thuras P, Mitchell JE: Subtypes of binge eating disorder based on psychiatric history. Int J Eat Disord 2005, 38:273-276.

63. Peterson CB, Thuras P, Ackard DM, Mitchell JE, Berg K, Sandager N, Wonderlich SA, Pederson MW, Crow SJ: Personality dimensions in bulimia nervosa, binge eating disorder, and obesity. Compr Psychiatry 2010, $51: 31-36$.

doi:10.1186/1471-244X-14-165

Cite this article as: Baiano et al.: Exploring health-related quality of life in eating disorders by a cross-sectional study and a comprehensive review. BMC Psychiatry 2014 14:165. 